\title{
In-Hand Manipulation of Objects with Unknown Shapes
}

\author{
Silvia Cruciani, Hang Yin and Danica Kragic
}

\begin{abstract}
This work addresses the problem of changing grasp configurations on objects with an unknown shape through in-hand manipulation. Our approach leverages shape priors, learned as deep generative models, to infer novel object shapes from partial visual sensing. The Dexterous Manipulation Graph method is extended to build upon incremental data and account for estimation uncertainty in searching a sequence of manipulation actions. We show that our approach successfully solves in-hand manipulation tasks with unknown objects, and demonstrate the validity of these solutions with robot experiments.
\end{abstract}

\section{INTRODUCTION}

In-Hand manipulation of unknown objects is a particularly challenging instance of dexterous manipulation. The problems of addressing where to grasp for given tasks and how to adapt the grasp on novel objects have been addressed and studied [1]-[3], but the problem of how to reach such a grasp starting from an initial one, without accessing the object's model, is still an open challenge. Reactive controllers accommodate local shape uncertainties via motion compliance and feedback, often relying on strong assumptions (e.g. invariant contact points [4]) or explorative trials [5], without aiming at a specific goal grasp. We target general in-hand manipulation to achieve a desired grasp on objects with unknown shapes. This calls for more flexible ways to handle unknowns and the capability of planning multi-stage actions as in Dexterous Manipulation Graphs (DMG) [6].

In contexts where the desired grasp is not directly reachable, the robot must adjust the object's configuration inside the hand after an initial grasp [7], [8]. In-hand manipulation solutions rely on accurate knowledge of geometry and dynamics of the specific grasped object [9], [10], or on many data samples [11], [12]. However, target grasp configurations can be obtained for a given task or object type [1], but do not strictly depend on the particular object shape (e.g. grasp by the handle). Instead of requiring a full object model, our method can operate on the object class level. We aim at providing an initial step towards successful integration of inhand manipulation with task-based grasp planning methods that do not rely on full knowledge of the object's shape, so that robots can successfully manipulate previously unseen objects to execute different tasks.

In this paper, we focus on achieving in-hand manipulation with a parallel gripper. Given no dexterity, grasp reconfiguration can be achieved by leveraging extrinsic dexterity [13], i.e. the exploitation of supports external to the gripper, such

S. Cruciani, H. Yin and D. Kragic are with the Division of Robotics, Perception and Learning, EECS at KTH Royal Institute of Technology, Stockholm, Sweden. $\{$ cruciani, hyin, dani\}@kth. se

This work was supported by the Swedish Foundation for Strategic Research project GMT14-0082 FACT. as pushing against a second gripper or external contacts [6], [14]-[16] and motions due to gravity and inertial forces [17][19]. In our case, we consider manipulation motions in which the object is pushed against the second robot's gripper, as in [6]. Moreover, we address the problem of in-hand manipulation by only exploiting vision; while tactile sensing improves the performances of controlling the slippage between the gripper's finger and the object's surface, visual feedback is often enough to obtain a successful in-hand manipulation execution [6], [20], even without relying on accurate dynamic models [21], [22].

Vision information of objects is partial and subject to occlusions. Our method exploits generative models to estimate a full object shape. Hence, the solutions can be searched for in an informative manner. Generative models build on a parameterized space to encode plausible object shapes. This has drawn much research attention in computer vision and graphics [23], [24]. Progress in deep generative models has enabled 3D shape synthesis in the form of voxels [25], point clouds [26] and implicit function representation [27]. Shape reconstruction has also been demonstrated in some of the works above. However, its application in manipulation is relatively recent [3], and targets transferring static grasps.

We extend the DMG method introduced in [6]. This method proposes to represent the object as a graph structure. The main disadvantages of DMG are that it needs a full model of the object shape and it requires an offline step for precomputing the graph. Once the graph is generated, it is used to plan any in-hand manipulation task on the given object. We propose a method that focuses on achieving a given in-hand manipulation task by exploiting only the information available to the robot when the task is given. Moreover, while in-hand manipulation is planned, we generate the graph so that successful manipulation tasks with the same object can re-use it, having the same advantages of DMG.

This work builds on our work in [6], where we introduce the idea of DMG. Here, we extend the work to consider objects of unknown shapes. We improve the DMG to represent the object reconstruction uncertainty and consider this uncertainty in the provided in-hand manipulation solution. The method presented here estimates DMG online and graph edges are added in relation to push feasibility.

\section{OVERVIEW}

\section{A. Preliminaries}

We represent a grasp configuration $G$ as a tuple: $\left\langle\mathbf{g}_{1}, \mathbf{g}_{2}, \mathcal{Q}\right\rangle . \mathbf{g}_{1}, \mathbf{g}_{2} \in \mathbb{R}^{3}$ are the two gripper's fingertips, in contact with the object, and $\mathcal{Q}$ is the unit quaternion representing the gripper's orientation. 
Since the object's model is unknown, these quantities cannot be expressed w.r.t. its reference frame $\Sigma_{o} \in S E(3)$. We express all the quantities in the initial gripper's frame $\Sigma_{g} . \Sigma_{o}$ and $\Sigma_{g}$ are rigidly attached, so a relative change in the grasp, i.e. a new gripper frame $\Sigma_{g}^{\prime}$ and a new grasp $G^{\prime}$, can be equivalently expressed in both of them.

The visible part of the object is a point cloud $P_{v} \subset \mathbb{R}^{3}$, i.e. a discrete set of $3 \mathrm{D}$ points. The reconstructed object, which contains the invisible part, is represented as another point cloud $P_{r} \subset \mathbb{R}^{3}$. The full object shape is the point cloud $P_{o}=P_{v} \cup P_{r}$. By $|P|$ we indicate the number of points in a point cloud.

To move the object within the gripper, we use the same in-hand movements as [6]: rotation and translation. A rotation $\phi \in(-2 \pi, 2 \pi)$ moves the grasp from $G=\left\langle\mathbf{g}_{1}, \mathbf{g}_{2}, \mathcal{Q}\right\rangle$ to $G^{\prime}=\left\langle\mathbf{g}_{1}, \mathbf{g}_{2}, \mathcal{Q}^{\prime}\right\rangle$. Due to the parallel gripper's structure, rotations about the fingertips are planar. A translation $\mathbf{t} \in$ $\mathbb{R}^{3}$ moves from $G=\left\langle\mathbf{g}_{1}, \mathbf{g}_{2}, \mathcal{Q}\right\rangle$ to $G^{\prime}=\left\langle\mathbf{g}_{1}^{\prime}, \mathbf{g}_{2}^{\prime}, \mathcal{Q}\right\rangle$. These movements can be executed by pushing the object against external fixtures or an additional gripper. Combinations of the two movement types at the same time are not considered during planning, but can occur during execution and will be compensated by a controller, as in [6].

We use $\mathcal{M}$ to denote a sequence of in-hand movements, e.g. $\phi_{0}, \mathbf{t}_{0}, \mathbf{t}_{1}, \phi_{1}, \ldots \phi_{K}$. This sequence is composed of $K$ rotations and $H$ translations.

A Dexterous Manipulation Graph $\mathcal{G}$ is a disconnected graph composed of a set of nodes $N_{\mathcal{G}}$ and a set of edges $E_{\mathcal{G}}$. A node $n \in N_{\mathcal{G}}$ is a tuple $\langle\mathbf{p}, \Theta\rangle ; \mathbf{p} \in \mathbb{R}^{3}$ represents a contact point between one fingertip and the object, and $\Theta \subseteq[0,2 \pi)$ is a continuous set of orientations w.r.t. the axis between the two fingertips that the gripper's finger can assume when in contact at p. Therefore, a single contact can correspond to more nodes, depending on the object's shape. Note that a DMG node represents a single finger, while for planning the two opposite gripper's fingers must be considered. An edge $e_{n_{a}, n_{b}} \in E_{\mathcal{G}}$ connects two nodes $n_{a}$, $n_{b}$ if: 1) it is possible for a fingertip to slide along the object from $\mathbf{p}_{a}$ to $\mathbf{p}_{b}$;2) $\Theta_{a} \cap \Theta_{b} \neq \emptyset$. As it is a disconnected graph, the DMG is composed of several components $C \subseteq E_{\mathcal{G}}$, that contain only the nodes connected to each other.

\section{B. Problem Statement and System Overview}

The in-hand manipulation problem is stated as: find the sequence of motions $\mathcal{M}$ that moves the gripper from $G_{i}$ to $G_{d}$, given the initial grasp $G_{i}=\left\langle\mathbf{g}_{i 1}, \mathbf{g}_{i 2}, \mathcal{Q}_{i}\right\rangle$, the desired grasp $G_{d}=\left\langle\mathbf{g}_{d 1}, \mathbf{g}_{d 2}, \mathcal{Q}_{d}\right\rangle$ and the object shape $P_{o}$.

We generate and use a partial Dexterous Manipulation Graph $\mathcal{G}$ that can be exploited for current and future in-hand manipulation tasks with the given object.

Our proposed method contains the following components:

1) Estimate the missing object shape $P_{r}$.

2) Build a partial DMG $\mathcal{G}$.

3) Plan motions $\mathcal{M}$ to move from $G_{i}$ to $G_{d}$.

We discuss all of them in detail in the next sections.

\section{Shape RECONSTRUCTION}

In this section, we present the steps of estimating the full object shape $P_{o}$ from a partial observation $P_{v}$. Simply registering the observed point cloud is inadequate in our case, since no object model is initially known. The idea is to exploit a shape prior which encapsulates a family of objects, e.g., hammers with variations on head and handle geometries. The prior can then be reasoned to complement $P_{v}$, in a similar way as humans imagine invisible parts by exploiting knowledge about the shapes of a certain type of object.

We propose to learn this shape prior as a probabilistic generative model, a denoising version of Variational Autoencoders (VAE) [28], from a set of object point clouds. The model takes a PointNet encoder [29] and a decoder with coarse intermediate reconstruction [30]. The shape prior is captured as an isotropic Gaussian $p(\mathbf{z}), \mathbf{z} \in \mathbb{R}^{d_{z}}, d_{z} \ll\left|P_{v}\right|$, in the latent space. Similar to [31], the full shape is recovered by searching $\mathbf{z}$ given the partial observation $P_{v}$ :

$$
\max _{\mathbf{z}} p\left(\mathbf{z} \mid P_{v}\right)=\min _{\mathbf{z}} \operatorname{Diff}\left(P_{v}, \operatorname{dec}(\mathbf{z})\right)-\beta \log p(\mathbf{z}),
$$

with $P_{r} \sim \operatorname{dec}\left(\mathbf{z}^{*}\right) . \beta$ denotes a weight balancing the reconstruction error and the plausibility according to the shape prior. The error term measures the distinction between evident and estimated point clouds and here we use a oneway Chamfer distance.

Solving (1) requires a good initialization for the underlying nonconvex optimization. This can be obtained by encoding the partial observation $P_{v}$. In practice, the pose of $P_{v}$ might not be well aligned to training data so we also propose to search a rigid transformation $\mathbf{T} \in S E(3)$ such that the initialization yields good performance:

$$
\mathbf{T}^{*}=\underset{\mathbf{T}}{\operatorname{argmax}}\left[\left.\max _{\mathbf{z}} p\left(\mathbf{z} \mid \mathbf{T}\left(P_{v}\right)\right)\right|_{\mathbf{z}_{\text {init }} \sim \operatorname{enc}\left(z \mid \mathbf{T}\left(P_{v}\right)\right)}\right] .
$$

The inner maximization can be approximated by a few gradient steps starting from the parameterized initialization. Empirically we found an expansion at $\mathbf{z}_{\text {init }}$ itself is sufficient as a surrogate. The rotational part of $\mathbf{T}$ takes an axis-angle representation and further transforms $P_{v}$ through Rodrigues' formula. The main axes of $P_{v}$ are decided by running a PCA and all combinations of axis directions are tried to facilitate the search of $\mathbf{T}^{*}$.

We generate training data from YCB objects [32], e.g., hammer and spoon, which are different from our test ones. The dataset is augmented by applying scaling and small rotations along the main axes. Point clouds are down-sampled to have a consistent number of points. The proposed VAE learns with raw point cloud data since the encoder and Chamfer distance are permutation-invariant.

\section{ONLINE DEXTEROUS MANiPUlation GRAPH}

\section{A. DMG Variations}

The main differences from [6] are that the new DMG $\mathcal{G}$ is not generated in a separate offline step, and that it does not need the full object shape. Moreover, we change and improve the graph structure as detailed below. 
Instead of focusing on all the possible motions that a fingertip can achieve, we link motions to the feasibility of executing them. Since we address the in-hand manipulation through pushes of the object, motions of the fingertips depend on the possible pushes. In [6] two nodes can be connected despite no push can move the fingertip between them. The infeasible pushes must be carefully excluded at planning time. In this work, since we merge the steps of DMG generation and in-hand manipulation planning, we incorporate in $\mathcal{G}$ only feasible motions.

We propose to represent the new DMG $\mathcal{G}$ as a directed graph: in contrast with the DMG in [6], which is an undirected graph, the existence of an edge $e_{n_{a} n_{b}} \in E_{\mathcal{G}}$ does not imply the connection $e_{n_{b} n_{a}} \in E_{\mathcal{G}}$.

In addition, we aim at obtaining $\mathcal{G}$ also for objects whose shape is only partially known. We introduce the functions $f(\cdot): \mathbb{R}^{3} \rightarrow[0,1], g(\cdot): E_{\mathcal{G}} \rightarrow[0,1]$. These functions are associations of points and edges to their certainty. We will also write $f\left(n_{a}\right)$, with $n_{a} \in N_{\mathcal{G}}$, by which we mean $f\left(\mathbf{p}_{a}\right)$. The value depends on the position $\mathbf{p}_{a}$ : if $\mathbf{p}_{a} \in P_{v}, f\left(\mathbf{p}_{a}\right)$ will be high, because that part of the object has been observed; in contrast, if $\mathbf{p}_{a} \in P_{r}, f\left(\mathbf{p}_{a}\right)$ will be lower. We define $g\left(e_{n_{a} n_{b}}\right)=\alpha \frac{\left(f\left(n_{a}\right)+f\left(n_{b}\right)\right)}{2}+(1-\alpha) f\left(\mathbf{p}_{p}\right)$, where $\alpha \in[0,1]$ is a weighting factor and $\mathbf{p}_{p}$ is the push point associated to the movement defined by the edge.

The partial DMG $\mathcal{G}$ is generated while looking for a solution $\mathcal{M}$ to move from $G_{i}$ to $G_{d}$. The returned solution will be the one with the highest certainty, to reduce the influence of the missing object knowledge on the execution.

\section{B. DMG Generation}

The graph $\mathcal{G}$ and the solution $\mathcal{M}$ are generated at the same time. The precedence goes to finding $\mathcal{M}$, but $\mathcal{G}$ is generated to facilitate future manipulations on the same object. To generate the graph, we introduce the following parameters: branching factor $b_{e} \in \mathbb{N}$, i.e. maximum number of edges from a node; edge length $l_{e} \in \mathbb{R}^{+}$, i.e. approximate Euclidean distance between node contact points; component threshold $\zeta \in \mathbb{R}^{+}$; push point normal threshold $t_{\mathbf{n}_{p}} \in[0,1]$.

At the beginning, the robot is grasping the object with configuration $G_{i}$. We create two nodes associated with it: $n_{i 1}=\left\langle\mathbf{g}_{i 1}, \Theta_{i 1}\right\rangle, n_{i 2}=\left\langle\mathbf{g}_{i 2}, \Theta_{i 2}\right\rangle . \Theta_{i 1}, \Theta_{i 2}$ are obtained by checking the collisions with the finger and gripper when in contact at the given point, as in [6]. Similarly, $G_{g}$ is associated with the nodes $n_{d 1}, n_{d 2}$. Each node is associated to a different DMG component $C_{i 1}, C_{i 2}, C_{d 1}, C_{d 2}$. The goal is to expand from $n_{i 1}, n_{i 2}$ and add nodes and edges to $\mathcal{G}$, until connections are found so that we can verify $C_{i 1}=C_{d 1}$, $C_{i 2}=C_{d 2}$. In this way, a solution $\mathcal{M}$ can exist.

The nodes $n_{i 1}, n_{i 2}$ define a grasp line, and we set this grasp line as normal $\mathbf{n}_{g}$ to the sliding area, regardless of the estimated normals in $P_{o}$. We also impose $f\left(n_{i 1}\right)=f\left(n_{i 2}\right)=1$.

The generation procedure is shown in Algorithms 1 and 2, where some steps have been omitted for the sake of conciseness (e.g. when used for planning, the graph generation stops when the goal becomes reachable). The graph is built starting from expanding the nodes $n_{i 1}, n_{i 2}$ and proceeding in a FIFO

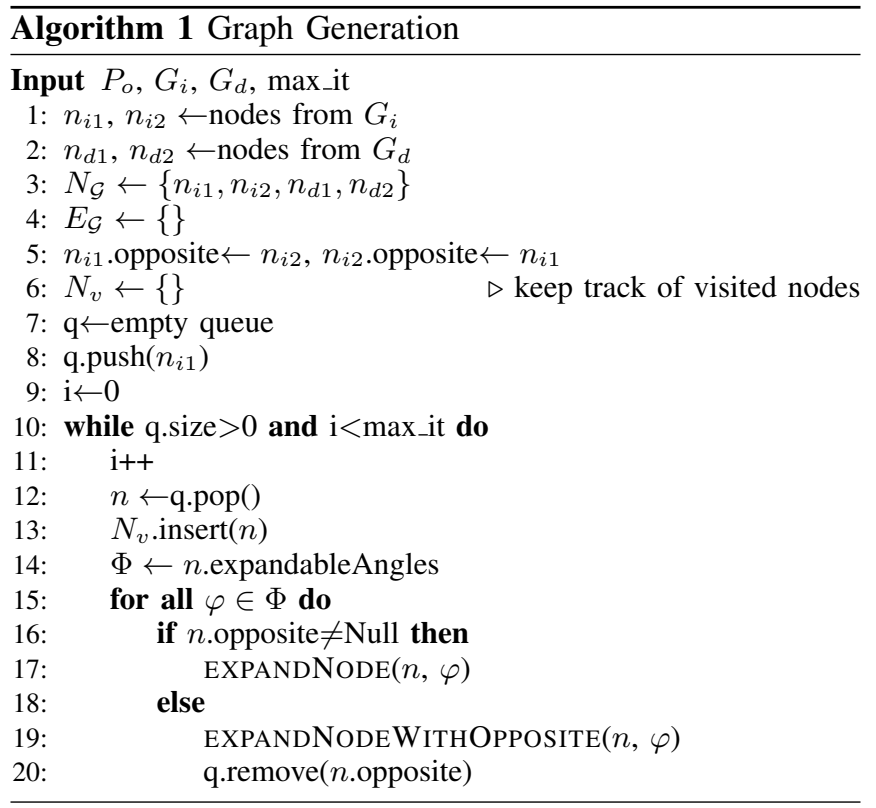

manner. We derive $b_{e}$ angles $\varphi_{1}, \ldots, \varphi_{b_{e}} \in \Phi \subset[0,2 \pi]$. These angles define directions on the object's surface and are used to expand the nodes and form new edges. The procedure of expanding a single node is detailed in section IV-E.

\section{Push Points}

We rely on pushes on the object to move it toward the desired pose. Hence, it is important to correctly identify a push point $\mathbf{p}_{p}$ that can be used for exerting the desired push. In this section, we focus on push points assuming translation motions. Similar processes are adopted for rotation, but with more relaxed constraints, as the push point and direction can be changed more freely without affecting the outcome.

A point $\mathbf{p}_{1} \in P_{o}$ can be associated with (at least) one opposite point $\mathbf{p}_{2} \in P_{o}$, found intersecting the object with the ray defined by $\mathbf{p}_{1}$ and the vector opposite to its normal to the surface. Given a translation motion $\mathbf{t}$ from $\mathbf{p}_{1}$, the corresponding $\mathbf{p}_{p} \in P_{o}$ is found by intersecting the object with the ray defined by $\frac{\mathbf{p}_{1}+\mathbf{p}_{2}}{2}$ and $-\mathbf{t}$. In case of multiple solutions, we choose $\mathbf{p}_{p}$ as the furthest away from $\mathbf{p}_{1}$.

To analyze feasible motions, we rely on the concept of motion cone [33], although we do not measure friction parameters and exploit a predefined tolerance. $\mathbf{t}$ is achievable if the normal to the object's surface $\mathbf{n}_{p}$ at the corresponding push point $\mathbf{p}_{p}$ is aligned with it. To consider the uncertainty in the object's shape, we verify it as

$$
\left|\mathbf{n}_{p} \cdot \frac{\mathbf{t}}{\|\mathbf{t}\|}\right| \geq \xi t_{\mathbf{n}_{p}},
$$

with $\xi \in[0,1]$ being a chosen adjustment for normal alignment depending on $f\left(\mathbf{p}_{p}\right)$. We prefer to relax the constraint when the object's shape is uncertain, and then correct this assumption afterwards rather than not considering the push admissible since the start. Uncertain pushes will be considered with lower priority, so that preferred pushes are those on the visible part of the object. 


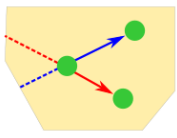

(a)

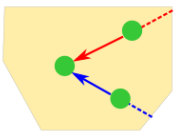

(b)

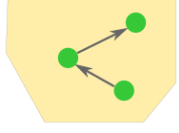

(c)
Fig. 1: Example of relation between graph edges and push directions.

Fig. 1a shows two examples of translations, and Fig. 1b shows the opposite motions. Feasible translations, considering the normal at the push point, are shown in blue; infeasible ones are in red. The constraint (3) results in a connectivity between those points in $\mathcal{G}$ as shown in Fig. 1c. Noticeably, motions are not necessarily reversible, which is why we changed the DMG structure to be a directed graph.

\section{DMG Components}

A component $C_{a} \subseteq N_{\mathcal{G}}$ contains all nodes between which it is possible for a single finger to slide or rotate without releasing the grasp. Due to the use of parallel grippers, motions are constrained to be locally planar along smooth surfaces. We associate a frame $\Sigma_{C_{a}}$ with each component. By convention, we assume that the $\mathrm{x}$-axis $\hat{\mathbf{x}}_{\Sigma_{C_{a}}}$ is aligned with the normal to the planar surface associated with the component. Given $n_{a} \in C_{a}$, a necessary condition for a neighbor node $n_{b}$ to belong to $C_{a}$ is

$$
\left|\hat{\mathbf{x}}_{\Sigma_{C_{a}}}^{T}\left(\mathbf{p}_{b}-\mathbf{p}_{a}\right)\right|<\zeta,
$$

where $\zeta \in \mathbb{R}^{+}$is a threshold to enable tolerance of slightly curved surfaces and account for noise in perception. The condition in (4) is not a sufficient condition, because a component must contain nodes that are connected. Note that the same point $\mathbf{p}_{a}$ can correspond to more nodes $n_{a 1}=\left\langle\mathbf{p}_{a}, \Theta_{a 1}\right\rangle, \ldots, n_{a M}=\left\langle\mathbf{p}_{a}, \Theta_{a M}\right\rangle$, and these nodes can belong to separate components. Similarly, certain translations might be infeasible due to (3) not being fulfilled.

\section{E. Node Expansion Step}

A node $n_{a}$ is associated with a set of expandable angles $\Phi_{a}$. For each $\varphi \in \Phi_{a}$, the expansion of $n_{a}$ is detailed in the following and summarized in Algorithm 2.

A new point $\mathbf{p}_{b}$ in $P_{o}$ is derived as

$$
\mathbf{p}_{b}=\underset{\mathbf{p} \in P_{o}}{\operatorname{argmin}}\left\|\mathbf{p}-\left(\mathbf{p}_{a}+l_{e} \mathbf{R}_{\hat{\mathbf{x}}_{\Sigma_{C}}}(\varphi) \hat{\mathbf{y}}_{\Sigma_{C_{a}}}\right)\right\|,
$$

where $\mathbf{R}_{\hat{\mathbf{x}}_{\Sigma_{C}}}(\varphi)$ is the axis-angle rotation matrix, and $\hat{\mathbf{y}}_{\Sigma_{C_{a}}}$ is the y-axis of frame $\Sigma_{C_{a}}$. If $\mathbf{p}_{b}$ does not satisfy (4), then no edge will be added. If there are no nodes in proximity of $\mathbf{p}_{b}$, then (at least) one new node $n_{b}$ is added to $N_{\mathcal{G}}$, associated with a new component $C_{b}$. If a finger in contact at $\mathbf{p}_{b}$ can rotate between $B$ disconnected angle sets $\Theta_{b 1}, \ldots \Theta_{b B}$, then $B$ disconnected nodes are added.

If (4) is satisfied, then the translation $\mathbf{t}=\mathbf{p}_{b}-\mathbf{p}_{a}$ from $\mathbf{p}_{a}$ is associated to the push point $\mathbf{p}_{p a}$. If (3) is fulfilled, then there is the chance to add new edges to the graph.

If $\mathbf{p}_{b}$ is close (i.e. within distance $\lambda$ ) to (at least) one existing node $n_{b}$, then for each node $n_{a}^{j}$ with position $\mathbf{p}_{a}$ an edge $e_{n_{a}^{j} n_{b}}$ is added to $E_{\mathcal{G}}$ only if $\Theta_{a}^{j} \cap \Theta_{b} \neq \emptyset$.

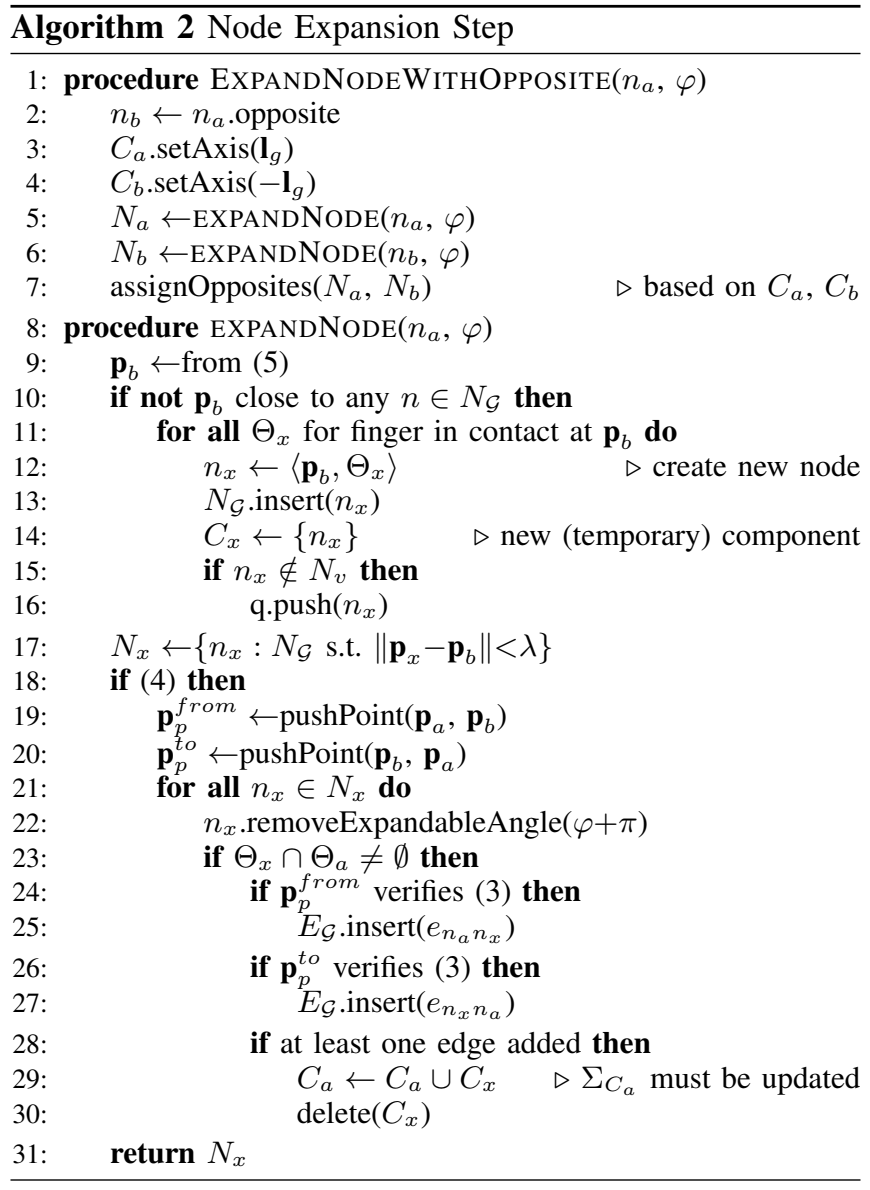

Notice that despite starting from $n_{a}$, other nodes can be expanded because to the same position $\mathbf{p}_{a}$ more nodes might correspond. If $C_{b} \neq C_{a}^{j}$, then we replace $C_{a}^{j}$ with $C_{a}^{j} \cup C_{b}$, and $C_{b}$ is deleted; the axes of the frame $\Sigma_{C_{a}^{j}}$ are updated based on all the new nodes to satisfy (4). If $\mathbf{p}_{b}$ is not in proximity of any node, then $J$ new nodes $n_{b}^{j}$ are added to $N_{\mathcal{G}}$, with $J$ the number of orientations $\Theta_{b}^{1}, \ldots, \Theta_{b}^{J}$. For each $n_{a}^{j}, e_{n_{a}^{j} n_{b}^{j}}$ is added to $E_{\mathcal{G}}$ and $n_{b}^{j}$ to $C_{a}^{j}$ only if $\Theta_{a}^{j} \cap \Theta_{b}^{j} \neq \emptyset$.

At the same time, the translation $-\mathbf{t}$ from $\mathbf{p}_{b}$ is associated with the push point $\mathbf{p}_{p b}$. If $\mathbf{p}_{p b}$ satisfies (3), then the edges towards $n_{a}$ are examined and added in the same way as the edges that depart from it.

Some nodes have an opposite node associated (e.g. $n_{i 1}$, $\left.n_{i 2}\right)$. In this case, the expansion is executed for both nodes at the same time, along the same direction. The vector $\hat{\mathbf{x}}_{\Sigma_{C}}$ is replaced by the one defined by the grasp line $\mathbf{l}_{g}=\frac{\mathbf{p}_{i 2}-\mathbf{p}_{i 1}}{\left\|\mathbf{p}_{i 2}-\mathbf{p}_{i 1}\right\|}$. If both components $C_{i 1}$ and $C_{i 2}$ are expanded, then the nodes keep carrying the opposite node with them, otherwise the expansion will be executed with only one node. See lines 1-7 in Algorithm 2.

When a node $n_{b}$ is generated or reached from an expansion obtained with angle $\varphi$, the opposite angle $\varphi^{\prime}$ is removed from $\Phi_{b} . \varphi^{\prime}$ is an approximation of $\varphi+\pi$. Therefore, $n_{b}$ will not be expanded along an area that has already been explored.

Fig. 2 shows a simple example of an expansion step. The initial $\mathcal{G}$ is shown in Fig. 2a. Each component corresponds to a different color. The node $n_{a} \in C_{3}$ is expanded in Fig. 2b, 


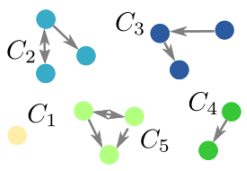

(a)

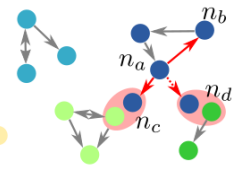

(b)

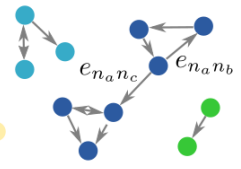

(c)
Fig. 2: Example of graph expansion step. Nodes of the same color belong to the same component.

into three points. The first one is associated with the node $n_{b}$. Since $n_{b} \in C_{3}, \Theta_{a} \cap \Theta_{b} \neq \emptyset$, and (3) is verified, the edge $e_{n_{a} n_{b}}$ is added to $\mathcal{G}$. The next point is close to $n_{c} \in C_{5} ;(3)$ is verified, and $n_{c}$ satisfies (4) for belonging to $C_{3}$; moreover, $\Theta_{a} \cap \Theta_{c} \neq \emptyset$. Therefore, $C_{3}$ is replaced with $C_{3} \cup C_{5}, C_{5}$ is removed from $\mathcal{G}$ and the edge $e_{n_{a} n_{c}}$ is added. Finally, the last point is close to the existing node $n_{d} \in C_{4}$. But, in this case, (3) is not verified, so no connection is added and no component is changed. The updated $\mathcal{G}$ is shown in Fig. 2c.

\section{F. In-Hand Manipulation Planning}

Once $\mathcal{G}$ is obtained by expanding nodes starting from $n_{i 1}$, $n_{i 2}$, it is used to plan in-hand manipulation. $\mathcal{G}$ is searched for a path from $n_{i 1}, n_{i 2}$ to $n_{d 1}, n_{d 2}$. We use the same Dijkstra procedure as [6] to account for both fingers moving at the same time on the object's surface, i.e. indicating a principal finger, and a secondary finger that follows it in the opposite node in $\mathcal{G}$ to ensure the validity of the whole path. However, we relax the constraint for the secondary node based on the value of $f$. For instance, a translation of the principal finger from $n_{a}$ to $n_{b}$ corresponds to a translation of the secondary finger from $n_{c}$ to $n_{d}$. If $C_{c} \neq C_{d}$, this solution should not be allowed, but we admit it if $f\left(n_{c}\right)$ and $f\left(n_{d}\right)$ are small, so that solutions are still considered and we account for possible errors in shape reconstruction.

Unlike in [6], the feasibility of pushes is already contained in $\mathcal{G}$, so it is not necessary to check it at planning time. Instead of obtaining the shortest path, we prefer to maximize the certainty of it, i.e. find the sequence of edges so that

$$
e_{n_{0} n_{1}}, \ldots, e_{n_{H-1} n_{H}}=\operatorname{argmax} \sum_{i=1}^{H} g\left(e_{n_{i-1} n_{i}}\right),
$$

where $n_{0}=n_{i 1}$ and $n_{H}=n_{d 1}$.

The solution from DMG is a path of nodes; this path has to be converted into $\mathcal{M}$ so that it can be executed. An edge $e_{n_{a} n_{b}} \in E_{\mathcal{G}}$ is easily converted into a translation $\mathbf{t}=\mathbf{p}_{b}-\mathbf{p}_{a}$, which can be executed by pushing against the corresponding push point. In contrast, rotations are not directly expressed in the DMG solution. We prefer to introduce rotations only when necessary, to minimize the amount of pushes the robot has to execute. Given two consecutive nodes $n_{a}, n_{b}$, and the gripper's finger in contact at $\mathbf{p}_{a}$ grasping with angle $\theta_{a}$, a rotation $\phi$ is introduces only if $\theta_{a} \notin \Theta_{b}$. A new angle $\theta_{b} \in \Theta_{a} \cap \Theta_{b}$ is chosen. We choose $\theta_{b}$ to minimize the amount of rotations in the overall path, but different options can be used (e.g. minimize $\left|\theta_{a}-\theta_{b}\right|$, reduce the distance from goal angle, etc).

\section{EXPERIMENTS}

\section{A. Building DMG Online}

In this section we evaluate our new DMG computation method and compare with the planning time of the offline DMG [6]. Because of this comparison, since the offline DMG uses the full object's shape, we used the full object point-cloud, using objects from the YCB set [32], and we disregard the object reconstruction for these results. The parameters of the offline DMG were: seed resolution 1.3 $\mathrm{cm}$, angle resolution $10^{\circ}$, normal threshold 0.17 . We refer to [6], [14] for details on these parameters and their effects on the solution. For our online DMG, we used the following parameters, chosen so that the graph outcome would be similar to the one of the offline DMG method in terms of connectivity and distance between node points: $l_{e}=0.012$, $b_{e}=5, t_{\mathbf{n}_{p}}=0.8, \xi=1, \zeta=0.002, \lambda=\frac{l_{e}}{2}$. We represented $\Theta$ with a discretization of $10^{\circ}$ to follow what was used in the offline DMG. The maximum iterations were 200, but we stopped the graph generation once the goal was reachable.

Fig. 3 shows a comparison in terms of time required for finding a solution for given in-hand manipulation tasks using two of the YCB objects: gelatin box and potted meat can. The offline DMG requires a first step to obtain the graph, which is then used for fast planning on the same object. In our online DMG method, the graph is built incrementally whenever a new task is added. We report times for finding solution for the tasks when no graph has been built (i.e. the object is seen for the first time), labeled online DMG from start. Compared to the offline DMG, these times are roughly 1 or 2 orders of magniture smaller.

Then, we show how building the DMG progressively reduces the required time for planning each task, because a partial graph is already available, by executing the tasks in sequence (online DMG 1-2-3 sequence).Moreover, when a task refers to nodes that have already been added to the graph, the planning time decreases even more, by 1 order of magnitude, as it is not necessary to expand the graph to explore not covered areas (online DMG 1-2-1 sequence).

The planning time of the offline DMG once the full DMG is built is still lower, but we believe this is due to the fact

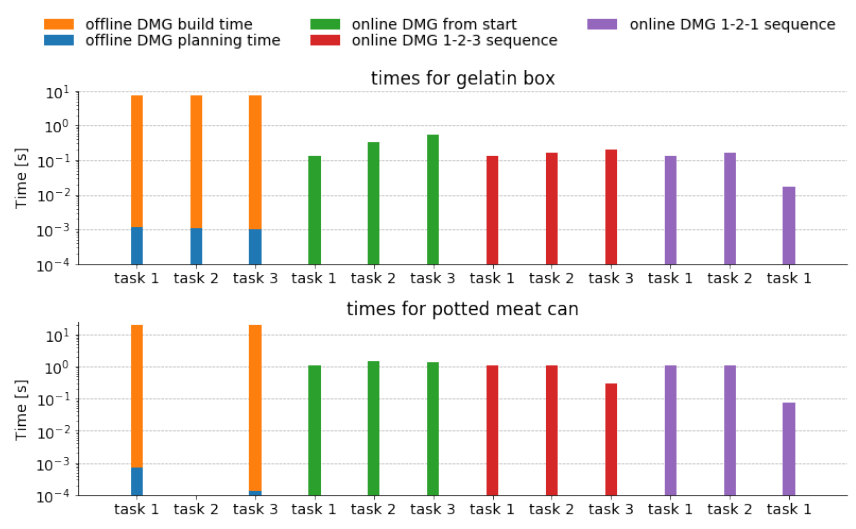

Fig. 3: The amount of time spent on a task, by the offline DMG [6] and by the proposed online DMG. For task 2 in potted meat can, the offline DMG failed to find a solution. The y axis is in logarithmic scale. 
TABLE I: Planned solution errors with full object shape

\begin{tabular}{lccc}
\hline & gelatin box & potted meat can & hammer \\
\hline $\operatorname{err}_{p}(\mathrm{~cm})$ & 0.56 & 1.01 & 0.52 \\
$\operatorname{err}_{o} \%$ & 3.2 & 4.8 & 4.8 \\
\hline
\end{tabular}

that the online method uses point clouds, while the offline method relies of the full object shape as a mesh, making it faster to check for the secondary finger's position during the graph search. However, we rely on the point cloud because this method is built to take into account direct sensory input.

Table I shows the average errors of 15 different tasks in the planned solution by online DMG for 3 of the YCB objects, obtained by simulating (kinematics only) reaching the desired grasps by using the found $\mathcal{M}$. Since in this case there is no reconstruction uncertainty, instead of using (6) to plan the solution, we use the shortest path criteria, as in [6].

The distance between $\mathbf{g}_{i_{1}}$ and $\mathbf{g}_{d_{1}}$ varied within 1.8 and $7.8 \mathrm{~cm}$ (5.9 in average). The desired rotation around the grasp line varied within $0^{\circ}$ and $90^{\circ} . \mathrm{err}_{p}$ is the average position error of the final contact points obtained applying the given translation sequence. $\operatorname{err}_{o} \%$ is the average error of orientations after applying the given rotations, obtained as norm of the difference of quaternions normalized to obtain the percentage w.r.t. the maximum possible orientation error [34]. For reference, an error of $90^{\circ}$ around a single axis wold result in $\mathrm{err}_{o} \%=54.1$.

Irregular and noisy object's shape increased the challenge in finding a good solution with the used parameters. A relaxation of thresholds might lead to better results when the object's reconstruction is noisy, and we use this strategy in the next section.

\section{B. DMG with Unknown Shapes}

In this section, we analyze the performance of our method including the shape reconstruction. We used a Kinect v2 sensor, mounted on top of an ABB Yumi robot, to collect the visible object $P_{v}$. We removed the robot's body from the depth image using [35]. We placed objects inside the robot's gripper and defined the desired grasp with respect to the initial grasp configuration. We used $\zeta=0.003, \xi=0.5$ if $f\left(\mathbf{p}_{p}\right)<0.9$, and 1 otherwise, and increased the maximum iterations to 300 to account for sensor noise and sparse reconstruction, $b_{e}=8, l_{e}=0.01$ and

$$
f(\mathbf{p})=\left\{\begin{array}{ll}
0.9 & \text { if } \mathbf{p} \in P_{v} \\
0.5 & \text { if } \mathbf{p} \in P_{r} \\
f\left(\tilde{\mathbf{p}}^{\star}\right) & \text { otherwise; } \tilde{\mathbf{p}}^{\star}=\underset{\tilde{\mathbf{p}} \in P_{o}}{\operatorname{argmin}}\|\mathbf{p}-\tilde{\mathbf{p}}\|_{2}
\end{array} .\right.
$$

We trained the VAE with point clouds from the YCB objects. On the test set of augmented data, the obtained reconstruction Chamfer error relative to three object dimensions were $0.78 \%, 2 \%$ and $7.8 \%$, respectively. In particular, for the presented experiments, we used two classes: hammer and spoon. Then, we run our in-hand manipulation method on objects grasped by the robot that were of the same kind but different shape from the objects present in the YCB set.
TABLE II: Planned solution errors with partial object shape

\begin{tabular}{lccc}
\hline & red hammer & black hammer & wooden spoon \\
\hline $\operatorname{err}_{p}(\mathrm{~cm})$ & 0.50 & 0.47 & 0.67 \\
$\operatorname{err}_{o} \%$ & 4.7 & 2.7 & 4.4 \\
no solution $\%$ & 13 & 20 & 12 \\
\hline
\end{tabular}

Table II shows the average errors over the different tasks in the planned in-hand manipulation solution when the object's shape is only partially visible. It also shows the times in which our method failed in finding a solution, although a solution exists. The distance from the initial to the desired grasp varied between 1.0 and $7.2 \mathrm{~cm}$. We noticed that the main challenge in this situation is due to the sparsity of $P_{r}$ with respect to $P_{v}$, which constitutes a source of error in the estimation of normals to the surface.

The higher failure rate for the black hammer with respect to the red hammer is likely due to a higher difference from the YCB hammer. Because of this difference, the reconstructed shape was not as close to the real object as it was for the red hammer, but it was still sufficient to provide good solution most of the times.

\section{Robot Experiments}

We executed the planned solution using the same control strategy of [6]. The pushes on the object were executed by using Yumi's second arm. The only difference from [6] is that in the dual-arm push we aligned the orientation of the pusher gripper's fingers (against which the object is pushed) with the desired translational motion, so that the side of the fingers in contact with the object's surface would be aligned with it, in order to minimize slippage and unwanted motions.

We used the same red hammer and corresponding tasks as in section V-B. We placed an April tag [36] on the object to measure the relative displacement between initial and final grasp frames $\Sigma_{g}, \Sigma_{g}^{\prime}$, and compare it with the desired change in the grasp. The average errors were $e r r_{p}=0.64 \mathrm{~cm}$ and $e r r_{o} \%=7.6$. In our experiments, the robot was able to adjust the object inside the gripper to the desired pose by using the planned in-hand path, converted into corresponding pushes against the object. In fact, despite slightly higher errors compared to the planned in-hand manipulation (Table II), the execution outcome can still be considered successful.

\section{CONClusions And Future WORK}

We presented an improvement over the Dexterous Manipulation Graph method for in-hand manipulation planning. The DMG can now take into account partial object shapes, it is generated progressively as manipulation tasks are given and takes into account the feasibility of the in-hand motions in the connections between nodes.

As future work, we plan to exploit the incorporation of uncertainty in the DMG to adapt the graph structure as new information on the object becomes available. In fact, after one or more pushes, more parts of the object shape become visible. This new information can be added to the graph by correcting the uncertain nodes and the uncertain edges. This will provide an instrument to adapt and improve the in-hand manipulation graph and the in-hand manipulation solution. 


\section{REFERENCES}

[1] M. Kokic, J. A. Stork, J. A. Haustein, and D. Kragic, "Affordance detection for task-specific grasping using deep learning," in 2017 IEEE-RAS 17th International Conference on Humanoid Robotics (Humanoids), Nov 2017, pp. 91-98.

[2] R. Antonova, M. Kokic, J. Stork, and D. Kragic, "Global search with bernoulli alternation kernel for task-oriented grasping informed by simulation," in Conference on Robot Learning, 2018.

[3] D. Rodriguez, C. Cogswell, S. Koo, and S. Behnke, "Transferring grasping skills to novel instances by latent space non-rigid registration," in IEEE Int. Conf. on Robotics and Automation (ICRA), 2018.

[4] Q. Li, C. Elbrechter, R. Haschke, and H. Ritter, "Integrating vision, haptics and proprioception into a feedback controller for in-hand manipulation of unknown objects," in 2013 IEEE/RSJ International Conference on Intelligent Robots and Systems, Nov 2013, pp. 24662471.

[5] H. van Hoof, T. Hermans, G. Neumann, and J. Peters, "Learning robot in-hand manipulation with tactile features," in 2015 IEEE-RAS 15th International Conference on Humanoid Robots (Humanoids), Nov 2015, pp. 121-127.

[6] S. Cruciani, C. Smith, D. Kragic, and K. Hang, "Dexterous manipulation graphs," in 2018 IEEE/RSJ International Conference on Intelligent Robots and Systems (IROS), Oct 2018, pp. 2040-2047.

[7] K. Hang, M. Li, J. A. Stork, Y. Bekiroglu, F. T. Pokorny, A. Billard, and D. Kragic, "Hierarchical fingertip space: A unified framework for grasp planning and in-hand grasp adaptation," IEEE Transactions on Robotics, vol. 32, no. 4, pp. 960-972, Aug 2016.

[8] B. Sundaralingam and T. Hermans, "Relaxed-rigidity constraints: Ingrasp manipulation using purely kinematic trajectory optimization," in Robotics: Science and Systems, 2017.

[9] R. Higo, Y. Yamakawa, T. Senoo, and M. Ishikawa, "Rubik's cube handling using a high-speed multi-fingered hand and a high-speed vision system," in 2018 IEEE/RSJ International Conference on Intelligent Robots and Systems (IROS), Oct 2018, pp. 6609-6614.

[10] N. C. Dafle, R. Holladay, and A. Rodriguez, "In-hand manipulation via motion cones," in Proceedings of Robotics: Science and Systems, Pittsburgh, Pennsylvania, June 2018.

[11] OpenAI, M. Andrychowicz, B. Baker, M. Chociej, R. Jozefowicz, B. McGrew, J. Pachocki, A. Petron, M. Plappert, G. Powell, A. Ray, J. Schneider, S. Sidor, J. Tobin, P. Welinder, L. Weng, and W. Zaremba, "Learning dexterous in-hand manipulation," 2018.

[12] V. Kumar, E. Todorov, and S. Levine, "Optimal control with learned local models: Application to dexterous manipulation," in 2016 IEEE International Conference on Robotics and Automation (ICRA), May 2016, pp. 378-383.

[13] N. C. Dafle, A. Rodriguez, R. Paolini, B. Tang, S. S. Srinivasa, M. Erdmann, M. T. Mason, I. Lundberg, H. Staab, and T. Fuhlbrigge, "Extrinsic dexterity: In-hand manipulation with external forces," in 2014 IEEE International Conference on Robotics and Automation (ICRA), May 2014, pp. 1578-1585.

[14] S. Cruciani, K. Hang, C. Smith, and D. Kragic, "Dual-arm in-hand manipulation and regrasping using dexterous manipulation graphs," 2019.

[15] N. Chavan-Dafle and A. Rodriguez, "Prehensile pushing: In-hand manipulation with push-primitives," in 2015 IEEE/RSJ International Conference on Intelligent Robots and Systems (IROS), Sep. 2015, pp. 6215-6222.

[16] D. L. Brock, "Enhancing the dexterity of a robot hand using controlled slip," in Proceedings. 1988 IEEE International Conference on Robotics and Automation, April 1988, pp. 249-251 vol.1.

[17] S. Cruciani and C. Smith, "In-hand manipulation using three-stages open loop pivoting," in 2017 IEEE/RSJ International Conference on Intelligent Robots and Systems (IROS), Sept 2017, pp. 1244-1251.

[18] J. Shi, J. Z. Woodruff, and K. M. Lynch, "Dynamic in-hand sliding manipulation," in Intelligent Robots and Systems (IROS), 2015 IEEE/RSJ International Conference on, Sept 2015, pp. 870-877.

[19] N. Chavan-Dafle and A. Rodriguez, "Sampling-based planning of inhand manipulation with external pushes," in International Symposium of Robotics Research, December 2017.

[20] F. E. Via B., Y. Karayiannidis, K. Pauwels, C. Smith, and D. Kragic, "In-hand manipulation using gravity and controlled slip," in 2015 IEEE/RSJ International Conference on Intelligent Robots and Systems (IROS), Sep. 2015, pp. 5636-5641.

[21] R. Antonova, S. Cruciani, C. Smith, and D. Kragic, "Reinforcement learning for pivoting task," arXiv preprint arXiv:1703.00472, 2017.
[22] B. Calli and A. M. Dollar, "Robust precision manipulation with simple process models using visual servoing techniques with disturbance rejection," IEEE Transactions on Automation Science and Engineering, vol. 16, no. 1, pp. 406-419, Jan 2019.

[23] N. Hasler, C. Stoll, M. Sunkel, B. Rosenhahn, and H.-P. Seidel, "A Statistical Model of Human Pose and Body Shape," Computer Graphics Forum, 2009.

[24] Q.-X. Huang, G.-X. Zhang, L. Gao, S.-M. Hu, A. Butscher, and L. Guibas, "An optimization approach for extracting and encoding consistent maps in a shape collection," ACM Trans. Graph. vol. 31, no. 6, pp. 167:1-167:11, Nov. 2012. [Online]. Available: http://doi.acm.org/10.1145/2366145.2366186

[25] J. Wu, C. Zhang, T. Xue, B. Freeman, and J. Tenenbaum, "Learning a probabilistic latent space of object shapes via $3 \mathrm{~d}$ generative-adversarial modeling," in Advances in Neural Information Processing Systems (NIPS), 2016, pp. 82-90.

[26] P. Achlioptas, O. Diamanti, I. Mitliagkas, and L. J. Guibas, "Learning representations and generative models for 3d point clouds," in Proceedings of the International Conference on Learning Representations (ICLR), 2017.

[27] J. J. Park, P. Florence, J. Straub, R. Newcombe, and S. Lovegrove, "Deepsdf: Learning continuous signed distance functions for shape representation," in The IEEE Conference on Computer Vision and Pattern Recognition (CVPR), June 2019.

[28] D. P. Kingma and M. Welling, "Stochastic gradient vb and the variational auto-encoder," in Proceedings of the International Conference on Learning Representations (ICLR), 2014.

[29] C. R. Qi, H. Su, K. Mo, and L. J. Guibas, "Pointnet: Deep learning on point sets for 3d classification and segmentation," Proc. Computer Vision and Pattern Recognition (CVPR), IEEE, 2017.

[30] W. Yuan, T. Khot, D. Held, C. Mertz, and M. Hebert, "Pcn: Point completion network," in 2018 International Conference on $3 D$ Vision (3DV), 2018, pp. 728-737.

[31] H. Yin, F. S. Melo, A. Billard, and A. Paiva, "Associate latent encodings in learning from demonstrations," in Proceedings of the National Conference on Artificial Intelligence (AAAI), San Francisco, USA, 2017.

[32] B. Calli, A. Singh, J. Bruce, A. Walsman, K. Konolige, S. Srinivasa, P. Abbeel, and A. M. Dollar, "Yale-cmu-berkeley dataset for robotic manipulation research," The International Journal of Robotics Research, vol. 36, no. 3, pp. 261-268, 2017.

[33] M. T. Mason, "Mechanics and planning of manipulator pushing operations," The International Journal of Robotics Research, vol. 5 , no. 3, pp. 53-71, 1986

[34] D. Q. Huynh, "Metrics for 3d rotations: Comparison and analysis," Journal of Mathematical Imaging and Vision, vol. 35, no. 2, pp. 155164 , Oct 2009

[35] [Online]. Available: https://github.com/blodow/realtime_urdf_filter

[36] E. Olson, "AprilTag: A robust and flexible visual fiducial system," in Proceedings of the IEEE International Conference on Robotics and Automation (ICRA). IEEE, May 2011, pp. 3400-3407. 